\title{
An Integral Equation Related to the Exterior Riemann- Hilbert Problem on Region with Corners
}

\author{
Zamzana Zamzamir $^{1, *}$, Munira Ismail ${ }^{2}$, Ali H. M. Murid ${ }^{2}$ \\ ${ }^{1}$ Department of Mathematics, Faculty of Science and Technology, Universiti Pendidikan Sultan Idris, 35900 Tg. Malim, Perak, \\ Malaysia \\ ${ }^{2}$ Department of Mathematics, Faculty of Science, Universiti Teknologi Malaysia, 81310 UTM, Skudai, Johor, Malaysia \\ *To whom correspondence should be addressed. E-mail: zamirzana@yahoo.com
}

Received: 31 October 2008

http://dx.doi.org/10.11113/mjfas.v4n2.45

\section{ABSTRACT}

Nasser in $\mathbf{2 0 0 5}$ gives the first full method for solving the Riemann-Hilbert problem (briefly the RH problem) for smooth arbitrary simply connected region for general indices via boundary integral equation. However, his treatment of RH problem does not include regions with corners. Later, Ismail in 2007 provides a numerical solution of the interior $\mathbf{R H}$ problem on region with corners via Nasser's method together with Swarztrauber's approach, but Ismail does not develop any integral equation related to exterior RH problem on region with corners. In this paper, we introduce a new integral equation related to the exterior RH problem in a simply connected region bounded by curves having a finite number of corners in the complex plane. We obtain a new integral equation that adopts Ismail's method which does not involve conformal mapping. This result is a generalization of the integral equation developed by Nasser for the exterior $\mathrm{RH}$ problem on smooth region. The solvability of the integral equation in accordance with the Fredholm alternative theorem is presented. The proof of the equivalence of our integral equation to the RH problem is also provided.

| Riemann-Hilbert problem | Fredholm integral equation |

\section{Introduction}

Many problems encountered in potential theory, the theory of elasticity, hydromechanics and in other branches of mathematical physics, can be modelled as boundary value problems of functions of complex variables. Some well-known complex boundary value problems are the Dirichlet problem, RH problem and Hilbert problem. They are generally treated in the context of singular integral equations which pose a great challenge for its numerical solution [1]. They are often solved by conformal mapping of the region to the unit disc, where the problems can be solved in closed form by harmonic conjugation [2].

Gakhov reduced the RH problem to three Dirichlet problems on the same region with a "regularizing factor" [3]. Murid and Nasser [4] have shown that RH problems can be treated with Fredholm integral equations of the second kind with continuous kernel, called generalized Neumann kernel. This is convenient for numerical calculations. Murid and Nasser's work is actually solving interior and exterior RH problem in smooth simply or multiply connected region. 
There are not many methods for solving the RH problem on region with corners in the literature because during the past twenty years, most of the research on the RH problem on smooth regions was concentrated on the numerical solutions of the problem. However, Swarztrauber [5] derived an integral equation related to the interior Dirichlet problem for a region with corners and constructed a numerical technique to solve the problem using Picard iteration method.

Recently, Ismail [6], provided an effective boundary integral equation method for seeking numerical solution of the interior RH problem on a simply connected region with a finite number of corners. Ismail successfully adapted the Swarztrauber's approach [5] to derive an integral equation associated to the RH problem with corners and provided the proofs of the solvability and uniqueness of the integral equation and its equivalence to the $\mathrm{RH}$ problem [7, 8]. Her works essentially are extensions of the interior Dirichlet problem with corners and for interior RH problem on smooth region.

In this paper, we provide a new integral equation related to the exterior $\mathrm{RH}$ problem in a simply connected region having a finite number of corners. We obtained a Fredholm integral equation of the second kind with continuous kernel. In this work, we strictly avoid conformal mapping. Our result is a generalization of the integral equation developed by Nasser's method [9] for his exterior RH problem on smooth region. Next, we establish the solvability for that new integral equation by applying two lemmas obtained by Murid and Nasser [4] and Wegmann et al. [10]. The proof of the equivalence of our integral equation to the RH problem is also given.

The organization of this paper is as follows. In Section 2, we recall the definition of the interior and exterior $\mathrm{RH}$ problem, present some auxiliary materials and give some known facts about the solvability of the RH problem. In Section 3, we show how we construct our new integral equation for the exterior $\mathrm{RH}$ problem on region with corners, establish the solvability for that new integral equation and prove the equivalence of our integral equation to the RH problem. Finally, a short conclusion is given in Section 4.

\section{Preliminaries}

Generally, the RH problem is a complex boundary value problem whose boundary condition is a linear combination of the real and imaginary parts of the boundary values of the function $f(z)$ [2]. We define $\Gamma$ to be at least a piecewise smooth Jordan curve with a counter clockwise parameterization $\Gamma: t=t(\tau), 0 \leq \tau \leq \rho$; having a continuously turning tangent except possibly at a finite number of corners $t_{1}, t_{2}, \ldots, t_{n}$ in the complex plane $\mathbf{C}$, where the inclinations of the positive semi-tangents have jumps of $\alpha_{1}, \alpha_{2}, \ldots, \alpha_{n}$ respectively. The angle $\alpha_{i}$ is positive if the jumps is in the counter clockwise direction and negative otherwise. We also define $\beta(t)$ be the angle between the two tangents to the $\Gamma$ at the point $t$, measured on the interior of the $\Gamma$. We call $\beta(t)$ a local interior angle and assuming $\beta(t)$ as $\pi$ at any point $t$ where $\Gamma$ has a unique tangent, and $\beta_{k}=\pi-\alpha_{k}$ at $t=t_{k}$. Cusps will not be considered, and hence it will be assumed that $0<\beta(t)<2 \pi[1$, p. 428], [3, pp. 31-32], $[11$, p. 176].

Suppose that $\Omega^{+}$and $\Omega^{-}$are the interior and exterior of $\Gamma$, respectively, such that the origin of the coordinate system belongs to $\Omega^{+}$and $\infty$ belongs to $\Omega^{-}$. If a function $f(z)$ is defined in a region containing $\Gamma$, then the limiting values of the function $f(z)$ when the point $z$ tends to the point $t \in \Gamma$ from the interior or the exterior of $\Gamma$ will be denoted by $f^{+}(t)$ and $f^{-}(t)$, respectively. Both the bounded region $\Omega^{+}$and the unbounded region $\Omega^{-}$are simply connected regions. It is assumed that the simply connected region lies in the extended complex plane $\mathbf{C} \cup\{\infty\}$. Suppose that $a, b$ and $\gamma$ are three real-valued functions defined on $\Gamma$, all 
satisfying the Hölder condition (see [3, p. 5] for definition) and $a^{2}(t)+b^{2}(t) \neq 0$ for all $t \in \Gamma$. In this paper we shall assume that the functions $a$ and $b$ have continuous first order derivative. Then the interior and exterior $\mathrm{RH}$ problems are defined as follows [2, p. 126].

Definition 2.1 The interior $R H$ problem in the bounded simply connected region $\Omega^{+}$consists of finding all functions $f(z)=u(z)+i v(z)$ that are analytic in $\Omega^{+}$, Hölder continuous on $\overline{\Omega^{+}}$, and satisfy the boundary condition

$$
a(t) u^{+}(t)-b(t) v^{+}(t)=\gamma(t)
$$

Another frequently used form of writing the boundary condition (1) is

$$
\operatorname{Re}\left[c(t) f^{+}(t)\right]=\gamma(t), \quad t \in \Gamma
$$

or equivalently as

$$
f^{+}(t)=-\frac{\overline{c(t)}}{c(t)} \overline{f^{+}(t)}+\frac{2 \gamma(t)}{c(t)}
$$

where

$$
c(t)=a(t)+i b(t), \quad t \in \Gamma .
$$

If $\gamma(t) \neq 0$, then (2) is a non-homogeneous boundary condition, while if $\gamma(t)=0$ we have a homogeneous boundary condition, i.e.,

$$
\operatorname{Re}\left[c(t) f^{+}(t)\right]=0, t \in \Gamma
$$

So, we call (2) as the non-homogeneous interior RH problem and (5) as the homogeneous interior RH problem.

Definition 2.2 The exterior RH problem in the unbounded simply connected region $\Omega^{-}$consists of finding all functions $f(z)=u(z)+i v(z)$ that are analytic in $\Omega^{-}$, vanish at $z=\infty$, Hölder continuous on $\overline{\Omega^{-}}$, and satisfy the boundary condition

$$
\operatorname{Re}\left[c(t) f^{-}(t)\right]=\gamma(t), \quad t \in \Gamma .
$$

The boundary condition (6) can be written in the equivalent form

$$
f^{-}(t)=-\frac{\overline{c(t)}}{c(t)} \overline{f^{-}(t)}+\frac{2 \gamma(t)}{c(t)}, \quad t \in \Gamma
$$

The homogeneous boundary condition of the exterior RH problem is given by

$$
\operatorname{Re}\left[c(t) f^{-}(t)\right]=0, t \in \Gamma \text {. }
$$


We call (6) as the non-homogeneous exterior RH problem and (8) as the homogeneous exterior RH problem. A more general exterior RH problem may be formulated by allowing its solution $f(z)$ to have polynomial growth at $z=\infty$.

\subsection{The Sokhotski Formulas}

For an integration contour having corner points, the limiting values $f^{+}(t)$ and $f^{-}(t)$ at all $t \in \Gamma$ for the Cauchy type integral

$$
f(z)=\frac{1}{2 \pi i} \int_{\Gamma} \frac{h(w)}{w-z} d w
$$

yield the Sokhotski formulas [3, p. 32],

$$
f^{+}(t)=\left(1-\frac{\beta(t)}{2 \pi}\right) h(t)+\frac{1}{2 \pi i} \int \frac{h(w)}{w-t} d w,
$$

and

$$
f^{-}(t)=-\frac{\beta(t)}{2 \pi} h(t)+\frac{1}{2 \pi i} \int \frac{h(w)}{w-t} d w
$$

Subtracting the formulas (10) and (11), we obtain for all $t \in \Gamma$ the following jump relation

$$
f^{+}(t)-f^{-}(t)=h(t)
$$

\subsection{The Fredholm Alternative Theorem}

Consider the Fredholm integral equation of the second kind

$$
\phi(x)-\lambda \int_{a}^{b} k(x, y) \phi(y) d y=g(x), \quad a \leq x \leq b,
$$

where $a$ and $b$ are finite, $k$ is a given real continuous function of two real variables, $g$ is a given real continuous function, $\lambda$ is a real parameter and $\phi$ is to be determined [3]. We state the Fredholm first theorem as follow.

Theorem 2.1 If $\lambda=\lambda_{0}$ is not an eigenvalue of the kernel $k$, then the integral equation (13) is uniquely solvable for an arbitrary right-hand side $g(x)$.

\subsection{Solvability of the RH Problem}

The solvability of the RH problem in a region $\Omega$ depends upon the geometry of the region $\Omega$ (bounded or unbounded, simply connected or multiply connected) as well as upon the index of the function $c(t)$ with respect to the boundary $\Gamma$, for definition of the index see [3, pp. 85-89]. The following lemma from Gakhov [3, p. 222; p. 226] shows the relation between the solvability of the RH problem and the index $x=i n d_{\Gamma}(c)$ of the function $c(t)$ which is also regarded as the index of the RH problem. 
Lemma 2.1 In the case $x \leq 0$, the homogeneous interior $R H$ problem (5) in the bounded simply connected region $\Omega^{+}$has $-2 x+1$ linearly independent solutions and the non-homogeneous problem (2) is solvable and its solution depends linearly on $-2 x+1$ arbitrary real constants. In the case $x>0$, the homogeneous problem has only the trivial solution and the non-homogeneous problem is solvable only if $2 x-1$ conditions are satisfied. If the latter conditions are satisfied, the non-homogeneous problem has a unique solution.

Lemma 2.2 In the case $x>0$, the homogeneous exterior RH problem (8) in the unbounded simply connected region $\Omega^{-}$has $2 x-1$ linearly independent solutions and the non-homogeneous problem (6) is solvable and its solution depends linearly on $2 x-1$ arbitrary real constants. In the case $x \leq 0$, the homogeneous problem has only the trivial solution and the non-homogeneous problem is solvable only if $-2 x+1$ conditions are satisfied. If the latter conditions are satisfied, then the non-homogeneous problem has a unique solution.

Next, we state two lemmas obtained by Murid and Nasser [4] and Wegmann et al. [10].

Lemma 2.3 If $x \leq 0$, then $\lambda=-1$ is not an eigenvalue of $N(c)$.

Lemma 2.4 If $x>0$, then $\lambda=-1$ is an eigenvalue of $N(c)$ with $2 x-1$ corresponding eigenfunctions.

The generalized Neumann kernel $N(c)$ will play an important role in this research. The solvability of the integral equations that we shall derive in this research for the exterior RH problem on region with corners depends on whether $\lambda= \pm 1$ are eigenvalues of $N(c)$ or not. Thus, in this section it is worth mentioning Nasser's integral equation with its kernel for exterior $\mathrm{RH}$ problem on smooth region. For convenience, we review this integral equation in the following theorem.

Theorem 2.2 Suppose $f(z)$ is any function analytic in $\Omega^{-}$of the exterior RH problem with smooth boundary $\Gamma$ satisfying boundary condition (6). If $\stackrel{\circ}{\mu}(t)=\operatorname{Im}\left[c(t) f^{-}(t)\right], t \in \Gamma$, then $\stackrel{\circ}{\mu}(t)$ satisfies the Fredholm integral equation

$$
\stackrel{\circ}{\mu}(t)-\int_{\Gamma} N(c)(t, w) \stackrel{\circ}{\mu}(w)|d w|=-\operatorname{Im}\left[c(t) L^{+}(t)\right], \quad t \in \Gamma
$$

where $N(c)(t, w)$ is the generalized Neumann kernel

$$
N(c)(t, w)=\left\{\begin{array}{cc}
\frac{1}{\pi} \operatorname{Im}\left[\frac{c(t)}{c(w)} \frac{T(w)}{w-t}\right] & w \neq t \in \Gamma, \\
\frac{1}{2 \pi\left|t^{\prime}(\tau)\right|} \operatorname{Im}\left[\frac{t^{\prime \prime}(\tau)}{t^{\prime}(\tau)}-\frac{2 c^{\prime}(t(\tau)) t^{\prime}(\tau)}{c(t(\tau))}\right] & w=t=t(\tau) \in \Gamma,
\end{array}\right.
$$

and

$$
L(z)=\frac{1}{2 \pi i} \int_{\Gamma} \frac{2 \gamma(t)}{c(t)} \frac{d t}{t-z}
$$

with $z \in \Omega^{-}$. 


\section{Results and Discussions}

Since in solving RH problem we are seeking the analytic function $f(z)$ in $\Omega^{-}$satisfying boundary condition (6), it is enough to find the real function $\mu(t)=\operatorname{Im}\left[c(t) f^{-}(t)\right], t \in \Gamma$, and then solve for $f^{-}(t)$ from $c(t) f^{-}(t)=\gamma(t)+i \stackrel{\circ}{\mu}(t)$. We have the following theorem stating our main result.

Theorem 3.1 Suppose that $\gamma(t)$ and $c(t)$ are specified by the exterior RH problem (6), and $\mu(t)$ is a realvalued function defined and satisfies the Hölder condition for all $t \in \Gamma$ and $f(z)$ is its solution. If

$$
\dot{\mu}(t)=\operatorname{Im}\left[c(t) f^{-}(t)\right], t \in \Gamma
$$

then $f(z)$ is given by

$$
f(z)=-\frac{1}{2 \pi i} \int_{\Gamma} \frac{\gamma(w)+i \mu(w)}{c(w)(w-z)} d w, \quad z \in \Omega^{-},
$$

whose boundary values are given by

$$
f^{-}(t)=\frac{\gamma(t)+i \mu(t)}{c(t)}, \quad t \in \Gamma,
$$

and furthermore,

$$
\frac{\gamma(t)+i \dot{\mu}(t)}{c(t)}=\frac{1}{[\beta(t)-2 \pi] i} \int_{\Gamma} \frac{\gamma(w)+i \stackrel{\circ}{\mu}(w)}{c(w)(w-t)} d w, \quad t \in \Gamma
$$

and $\mu(t)$ satisfies

$$
\dot{\mu}(t)-\frac{1}{\beta(t)-2 \pi} \int_{\Gamma} \dot{\mu}(w) \operatorname{Im}\left[\frac{c(t)}{c(w)} \frac{d w}{w-t}\right]=-\frac{1}{\beta(t)-2 \pi} \int_{\Gamma} \gamma(w) \operatorname{Re}\left[\frac{c(t)}{c(w)} \frac{d w}{w-t}\right] .
$$

Proof:

If $\mu(t)$ satisfies (17), then

$$
c(t) f^{-}(t)=\operatorname{Re}\left[c(t) f^{-}(t)\right]+i \operatorname{Im}\left[c(t) f^{-}(t)\right]=\gamma(t)+i \dot{\mu}(t) .
$$

Equation (22) implies that the boundary values $f^{-}(t)$ is given by (19) and in accordance with the Cauchy integral formula, $f(z)$ can be represented by (18).

Furthermore, $f(z)=f(\infty)=0$ when $z \in \Omega^{+}$implying that $f^{+}(t)=0$. Hence by the Sokhotski formula (10) we have

$$
0=\left(1-\frac{\beta(t)}{2 \pi}\right)\left(-\frac{\gamma(t)+i \dot{\mu}(t)}{c(t)}\right)+\frac{1}{2 \pi i} \int_{\Gamma}-\frac{\gamma(w)+i \dot{\mu}(w)}{c(w)(w-t)} d w, \quad t \in \Gamma
$$

which leads to (20). 
Next, multiplying both sides of (20) by $c(t)$ and taking its imaginary part gives

$$
\mu(t)=-\frac{1}{\beta(t)-2 \pi} \int_{\Gamma} \gamma(w) \operatorname{Re}\left[\frac{c(t)}{c(w)} \frac{d w}{w-t}\right]+\frac{1}{\beta(t)-2 \pi} \int_{\Gamma} \stackrel{\circ}{\mu}(w) \operatorname{Im}\left[\frac{c(t)}{c(w)} \frac{d w}{w-t}\right]
$$

which can be rewritten as (21).

The integral equation (21) is the integral equation related to the exterior $\mathrm{RH}$ problem (6). It is also a generalization of the integral equation (14) for the exterior RH problem on smooth region.

Before we establish the solvability of the integral equation (21), we need first to show it is a generalization of the integral equation (14) for the exterior RH problem on smooth region. We state that if $\Gamma$ smooth everywhere, then $\beta(t)$ is always $\pi$, and the kernel of integral equation (21) is also the generalized Neumann kernel of (14) except it is no longer compact because $\Gamma$ has a finite number of corners.

We show that the right-hand side of (21) is equivalent to the right-hand side of (14). Applying the Sokhotski formula (10) to (16) and multiplying the result with $c(t)$, we have

$$
c(t) L^{+}(t)=\frac{2 \pi-\beta(t)}{\pi} \gamma(t)-i \frac{1}{\pi} \int_{\Gamma} \frac{c(t)}{c(w)} \frac{\gamma(w)}{w-t} d w .
$$

Since $\gamma(t)$ is a real function then the imaginary part of $(25)$ gives

$$
\operatorname{Im}\left[c(t) L^{+}(t)\right]=-\frac{1}{\pi} \int_{\Gamma} \gamma(w) \operatorname{Re}\left[\frac{c(t)}{c(w)} \frac{d w}{w-t}\right]
$$

which is equivalent to the right-hand side of (21) when $\beta(t)$ is always $\pi$. This is equivalent everywhere except at the corners, hence proves that (21) is a generalization of (14).

Now we establish the solvability of the integral equation (21). For $\beta(t)=\pi,(21)$ becomes

$$
\dot{\mu}(t)+\int_{\Gamma} \frac{1}{\pi} \operatorname{Im}\left[\frac{c(t)}{c(w)} \frac{T(w)}{w-t}\right] \dot{\mu}(w)|d w|=\frac{1}{\pi} \int_{\Gamma} \gamma(w) \operatorname{Re}\left[\frac{c(t)}{c(w)} \frac{d w}{w-t}\right] .
$$

Comparing (27) with the Fredholm integral equation of the second kind (13), we will obtain $\lambda=-1$. According to Fredholm first theorem (see Theorem 2.1), if $\lambda=-1$ is not an eigenvalue of its kernel, then the integral equation (21) is uniquely solvable. The following theorem represents the solvability of our integral equation.

Theorem 3.2 If $x \leq 0$, then integral equation (21) is uniquely solvable. If $x>0$, then integral equation (21) is non-uniquely solvable.

Proof:

For the case $x \leq 0$, Lemma 2.3 implies that in accordance with the Fredholm alternative theorem, integral equation (21) is uniquely solvable. For the case $x>0$, Lemma 2.4 implies that in accordance with the Fredholm alternative theorem, integral equation (21) is always solvable and its general solution contains $2 x-1$ arbitrary constants; hence it is non-uniquely solvable. 
Theorem 3.1 states that if $f(z)$ given by (18) is a solution of the RH problem (6), then the real function $\mu(t)$ given by (17) is a solution of the integral equation (21). Our next task is to show the converse, i.e. if the RH problem (6) is solvable and $\mu(t)$ is a solution of the integral equation (21), then the function $f(z)$ defined by (18) is a solution of the RH problem (6). Thus, we have the following theorem to establish equivalence.

Theorem 3.3 Suppose the RH problem (6) is solvable. Further suppose $\gamma(t)$ and $c(t)$ are specified by (6), and $\dot{\mu}(t)$ is a real-valued function defined and satisfies the Hölder condition for all $t \in \Gamma$. If $\mu(t)$ is a solution of the integral equation (21), then it satisfies (17) and $f(z)$ given by (18) is the solution of (6).

Proof:

Suppose that the function $\mu(t)$ is a solution of the integral equation (21) and $f(z)$ be defined by (18) satisfying the Sokhotski formulas (10) and (11). Thus by Sokhotski formula (11), (18) becomes

$$
f^{-}(t)=\frac{\beta(t)}{2 \pi}\left(\frac{\gamma(t)+i \stackrel{\circ}{\mu}(t)}{c(t)}\right)-\frac{1}{2 \pi i} \int_{\Gamma} \frac{\gamma(w)+i \dot{\mu}(w)}{c(w)} \frac{d w}{w-t} .
$$

Multiplying both sides of (28) by $c(t)$ gives

$$
c(t) f^{-}(t)=\frac{\beta(t)}{2 \pi}(\gamma(t)+i \dot{\mu}(t))-\frac{1}{2 \pi i} \int_{\Gamma} \gamma(w)+i \dot{\mu}(w) \frac{c(t)}{c(w)} \frac{d w}{w-t} .
$$

Rewriting $\frac{c(t)}{c(w)} \frac{d w}{w-t}=A+i B$, the integral equation (21) becomes

$$
\dot{\mu}(t)=-\frac{1}{\beta(t)-2 \pi} \int_{\Gamma} \gamma(w) A-\dot{\mu}(w) B,
$$

or can be written as

$$
\int_{\Gamma}(\gamma(w) A-\ddot{\mu}(w) B)=-(\beta(t)-2 \pi) \stackrel{\circ}{\mu}(t) .
$$

Equation (29) can also be written in terms of $A \& B$ as

$$
\begin{aligned}
c(t) f^{-}(t) & =\frac{\beta(t)}{2 \pi}(\gamma(t)+i \dot{\mu}(t))+\frac{i}{2 \pi} \int_{\Gamma}[(\gamma(w) A-\mu(w) B)+i(\gamma(w) B+\mu(w) A)], \\
& =\frac{\beta(t)}{2 \pi}(\gamma(t)+i \dot{\mu}(t))+\frac{i}{2 \pi} \int_{\Gamma}(\gamma(w) A-\dot{\mu}(w) B)-\frac{1}{2 \pi} \int_{\Gamma}(\dot{\mu}(w) A+\gamma(w) B) .
\end{aligned}
$$

Substituting (31) into (32) and simplifying gives

$$
c(t) f^{-}(t)=i \stackrel{\circ}{\mu}(t)+\frac{\beta(t)}{2 \pi} \gamma(t)-\frac{1}{2 \pi} \int_{\Gamma}(\stackrel{\circ}{\mu}(w) A+\gamma(w) B) .
$$


The imaginary part of (33) proves that $\mu(t)$ also satisfies

$$
\operatorname{Im}\left[c(t) f^{-}(t)\right]=\stackrel{\circ}{\mu}(t) .
$$

By formula (12), we have

$$
f^{+}(t)-f^{-}(t)=-\frac{\gamma(t)+i \mu(t)}{c(t)},
$$

which upon multiplying both sides by $c(t)$ gives

$$
c(t) f^{+}(t)-c(t) f^{-}(t)=-\gamma(t)-i \mu(t) \text {. }
$$

The imaginary part of (36) is

$$
\operatorname{Im}\left[c(t) f^{+}(t)-c(t) f^{-}(t)\right]=-\mu(t)
$$

and due to (34) implies that the integral equation (21) is equivalent to the interior RH problem

$$
\operatorname{Im}\left[c(t) f^{+}(t)\right]=0 .
$$

Equation (38) can be transformed into (8) by replacing $c(t)$ by $\tilde{c}(t)=i c(t)$. Equations (36) and (38) imply that the function $f(z)$ defined by (18) satisfies

$$
\operatorname{Re}\left[c(t) f^{-}(t)\right]-c(t) f^{+}(t)=\gamma(t)
$$

If $x>0$, then by Lemma 2.1, (38) has only the trivial solution hence $f^{+}(t)=0$ and substituting this into (39) implies that $f(z)$ satisfies the problem (6) and by (34) its boundary values are given by (19).

However, in the case of $x \leq 0$, by Lemma 2.2 the RH problem (6) may be unsolvable and it follows from Theorem 3.1 that the integral equation (21) has a solution when the problem is solvable, this implies that $f(z)$ defined by (18) satisfies (39) with $c(t) f^{+}(t)=0$, hence $f(z)$ satisfies the RH problem (6) and its boundary values are given by (19).

\section{Conclusion}

A boundary integral equation has been derived for the exterior $\mathrm{RH}$ problem on region with corners. The approach of deriving the integral equation is the same as how Ismail [6] derived her integral equation associated with the interior RH problem on region with corners. Also in this paper, the new integral equation was shown to be a generalization of Nasser's integral equation associated with the exterior RH problem on arbitrary simply connected smooth region followed by the establishment of the solvability and the uniqueness of the derived integral equation and its equivalent to the $\mathrm{RH}$ problem.

\section{Acknowledgements}

The first author is a PhD student supported by Universiti Pendidikan Sultan Idris. The second and third authors were supported in part by the Malaysian Ministry of Higher Education (MOHE) through FRGS funding, Project Vote: 78270. This support is gratefully acknowledged. 


\section{References}

[1] N. I. Muskhelishvili, Singular Integral Equations, English translation of Russian edition 1953, Leyden: Noordhoff, 1977.

[2] P. Henrici, Applied and Computational Complex Analysis, Vol. 3, New York: John Wiley, 1986.

[3] F. D. Gakhov, Boundary Value Problem, English translation of Russian edition 1963, Oxford: Pergamon Press, 1966.

[4] A. H. M. Murid and M. M. S. Nasser, Bull. Malaysian Math. Sc. Soc. (Second Series), 26 (2003) 13-33.

[5] P. N. Swarztrauber, SIAM Journal of Numerical Analysis, 9 (1972) 300-306.

[6] M. Ismail, Numerical Solution of the Interior Riemann-Hilbert Problem on Region with Corners via Boundary Integral Equation, Ph.D. Thesis, Universiti Teknologi Malaysia, 2007.

[7] M. Ismail, A. H. M. Murid and B. Sanugi, International Journal of Simulation And Process Modelling, Inderscience Enterprise Ltd., 2 (2006) 25-32.

[8] M. Ismail, B. Sanugi and A. H. M. Murid, International Journal of Pure and Applied Mathematics, Academic Publication, Bulgaria, 31 (2006) 379-400.

[9] M. M. S. Nasser, Boundary Integral Equation Approach for the Riemann Problem, Ph.D. Thesis, Universiti Teknologi Malaysia, 2005.

[10] R. Wegmann, A. H. M. Murid and M. M. S. Nasser, J. Comput. Appl. Math., 182 (2005) 388-415.

[11] E. Hille, Analytic Function Theory, Vol. 1, New York: Chelsea Publishing Company, 1973. 